\title{
REVIEW
}

\section{A Review of the Key Clinical Trials of 2015: Results and Implications}

\author{
Claire McCune $\cdot$ Peter McKavanagh · Ian B. A. Menown
}

Received: April 11, 2016/ Published online: June 8, 2016

(C) The Author(s) 2016. This article is published with open access at Springerlink.com

\section{ABSTRACT}

Introduction: Multiple significant, potentially practice changing clinical trials in cardiology have been conducted and subsequently presented throughout the past year.

Methods: In this paper, the authors have reviewed and contextualized significant cardiovascular clinical trials presented at major international conferences of 2015 including American College of Cardiology, European Association for Percutaneous Cardiovascular Interventions, American Diabetes Association, European Society of Cardiology, Transcatheter Cardiovascular Therapeutics, Heart Rhythm Congress, and the American Heart Association Scientific Sessions.

Results: The authors describe new trial data for heart failure (including eplerenone, finerenone, patiromer, sacubitril/valsartan, the beta 3 agonist mirabegron, sitagliptin, empagliflozin,

Enhanced content To view enhanced content for this article go to http://www.medengine.com/Redeem/83D4 F0606921672B.

C. McCune · P. McKavanagh · I. B. A. Menown ( $₫)$ Craigavon Cardiac Centre, Craigavon Hospital, Southern Trust, Craigavon BT63 5QQ, Northern Ireland, UK

e-mail: ian.menown@southerntrust.hscni.net alginate-hydrogel LV epicardial implant), anticoagulation (idarucizumab and andexanet alfa reversal agents, adherence programmes, practice in ablation), transcatheter aortic valve replacement (long-term data, valve-in-valve use, the TriGuard embolic deflecting device), patent foramen ovale closure, cardiovascular prevention (PCSK9 inhibitors, hypertension treatment) and antiplatelets strategies (extended duration therapy with clopidogrel or ticagrelor). Trial data are also described for contemporary technologies including the Biofreedom polymer-free drug coated stent, bioabsorbable stents, PCI strategies, left main treatment, atrial fibrillation ablation techniques, leadless pacemakers and the role of coronary computed tomographic angiography.

Conclusions: This paper summarizes and contextualizes multiple pertinent 2015 clinical trials and will be of interest to both clinicians and cardiology researchers.

Keywords: Acute coronary syndrome; Anticoagulation; Antiplatelet; Atrial fibrillation; Bioabsorbable polymer; Coronary 
prevention; Electrophysiology; Heart failure; Myocardial infarction; Pacemaker

\section{INTRODUCTION}

Over the past year, multiple noteworthy trials with the potential to evolve current clinical practice and guidelines have been presented at international meetings including the American College of Cardiology (ACC, San Diego, USA, March 2015), European Association for Percutaneous Cardiovascular Interventions (EuroPCR, Paris, France, May 2015), American Diabetes Association (ADA, MA, USA, June 2015), European Society of Cardiology (ESC, London, UK, August 2015), Transcatheter Cardiovascular Therapeutics (TCT, San Francisco, USA, October 2015), Heart Rhythm Congress (HRC, Birmingham, UK, October 2015), and the American Heart Association Scientific Sessions (AHA, Florida, USA, November 2015). In this paper the authors describe new clinical data placed in the context of acute coronary syndrome (ACS), interventional cardiology, heart failure, atrial fibrillation, electrophysiology, and coronary prevention.

\section{METHODS}

The results of major clinical trials in cardiology presented at major conferences in 2015 were reviewed by the authors. Search terms included "acute coronary syndrome", "atrial fibrillation", "coronary prevention", "electrophysiology", "heart failure", and "interventional cardiology". Trials were selected based on relevance to the cardiovascular (CV) community, the potential to change clinical practice, and the potential to guide further phase 3 research. This article is based on previously conducted studies and does not involve any new studies of human or animal subjects performed by any of the authors.

\section{HEART FAILURE}

Advances in heart failure (HF) have featured prominently in many 2015 conferences with particular focus on addressing the side effects of currently used drug classes. Mineralocorticoid antagonists (MRAs) have a class 1A recommendation for reduction in mortality and hospitalization in patients with heart failure with reduced ejection fraction (EF). However, this drug class is typically under-prescribed due to concerns regarding hyperkalemia and renal impairment in high-risk patients. Finerenone is a novel non-steroidal MRA with greater receptor selectivity than spironolactone and better receptor affinity than eplerenone in vitro $[1,2]$, which may have the potential to reduce side effects of hyperkalemia and renal impairment [1]. The ARTS-HF (mineralocorticoid Receptor antagonist Tolerability Study-Heart Failure) double-blind controlled phase 2b trial (ClinicalTrials.gov \#NCT01807221) randomized 1060 patients with heart failure (HF), type 2 diabetes mellitus $(\mathrm{T} 2 \mathrm{DM}) \pm$ chronic kidney disease (CKD) to finerenone (titrated from one of five doses from 2.5 to $20 \mathrm{mg}$ ) or eplerenone (titrating from 25 to $50 \mathrm{mg}$ ) [1, 2]. Although the proportion of patients with a relative decrease of $30 \%$ in 90-day NT-proBNP (primary endpoint) was similar in all groups, the measurement of NT-proBNP to predict clinical outcome is not well established, and the subgroup of patients receiving finerenone $10-20 \mathrm{mg}$ showed a $44 \%$ reduction the secondary clinical composite endpoint of 
90-day all-cause death, CV death, hospitalization, or emergency presentation for worsening chronic HF $[1,2]$. Finerenone 10-20 mg appeared better tolerated than eplerenone with a smaller increase in potassium (0.119 vs. $0.262 \mathrm{mmol} / \mathrm{l} ; p=0.016)$ and lower incidence of estimated glomerular filtration rate (eGFR) decrease $>40 \%$ (4.2\% vs. $9.4 \%)[1,2]$. A phase 3 trial has been announced.

Hyperkalemia is a common concern in HF patients using renin-angiotensin-aldosterone (RAAS) inhibitors [3]. In a previous trial, OPAL-HK (ClinicalTrials.gov \#NCT01810939), investigators showed a short-term sustained decrease in potassium in patients with $\mathrm{HF}$ taking the potassium binder patiromer with RAAS inhibitors [4]. The phase 2 AMETHYST DN trial (ClinicalTrials.gov \#NCT01371747) assigned 304 patients with CKD, hypertension (HTN), T2DM, serum potassium above $5 \mathrm{mEq} / \mathrm{l}$ and who were taking RAAS inhibitors to patiromer (starting dose stratified by baseline potassium) [5]. Patiromer reduced potassium within days and maintained levels $<5 \mathrm{mEq} / \mathrm{l}$ in patients with HF $(n=105)$ or without HF $(n=199)$ for up to 1 year $(p<0.001)$. In total, $74 \%$ of the HF subgroup completed the trial, $7 \%$ withdrew consent, and a further $7 \%$ had adverse effects such as diarrhea, hypokalemia, and hypomagnesemia [5]. Further studies are planned to assess if improved control of hyperkalemia will translate into survival benefit [5].

Renal dysfunction is another common issue with RAAS inhibitors. A combination of the neprilysin inhibitor sacubitril and the angiotensin receptor blocker valsartan (LCZ696) was previously shown to decrease the primary endpoint of $\mathrm{CV}$ death or $\mathrm{HF}$ hospitalization, CV death, and a reduction in all-cause mortality compared with enalapril in the 2014 PARADIGM-HF study (prospective comparison of ARNI with ACEI to Determine Impact on Global Mortality and Morbidity in HF) (ClinicalTrials.gov \#NCT01035255) [3, 6]. The renal tolerability of sacubitril/valsartan was presented at the ESC Congress 2015 [7]. Over a 48 -month period, eGFR decreased by $0.14 \mathrm{ml} /$ $\min / 1.73 \mathrm{~m}^{2}$ in the enalapril group and $0.11 \mathrm{ml} / \mathrm{min} / 1.73 \mathrm{~m}^{2}$ in the sacubitril/valsartan groups, respectively ( $p=0.001$ ) [7]. Although, it should be noted that patients with an acute initial decline in eGFR were excluded from the PARADIGM-HF study [7], CV death or HF hospitalization was reduced similarly in the CKD group (36\% of the study population) compared to those without baseline CKD (HR 0.790 vs. $0.799 ; p=0.90$ for interaction), [7] suggesting a favorable cardiorenal profile [7].

The value of MRA treatment in mild heart failure has already been established by the prior EMPHASIS-HF study (Eplerenone in Mild Patients Hospitalization and Survival Study in Heart Failure) (ClinicalTrials.gov \#NCT00232180) in which eplerenone reduced the rate of $\mathrm{CV}$ death or $\mathrm{HF}$ hospitalization (18.3\% vs. 25.9\%; HR 0.63) among patients with New York Heart Association (NYHA) class II symptoms and an EF $\leq 35 \%$ [8]. The potential for extension of MRA use to patients without previously diagnosed HF was investigated by the open label ALBATROSS (Aldosterone Lethal Effects Blockade in Acute Myocardial Infarction Treated With or Without Reperfusion to Improve Outcome and Survival at Six Months' Follow-Up) (ClinicalTrials.gov \#NCT01059136) trial in which 1600 patients without known HF were randomized to spironolactone or control within $72 \mathrm{~h}$ of MI (myocardial infarction) [9]. Although there was a suggestion of benefit in the ST elevation subgroup (1229), overall, no significant difference was found in the primary composite endpoint of death, resuscitated 
cardiac death, ventricular fibrillation/ ventricular tachycardia, $\mathrm{HF}$, or indication for implantable cardiac defibrillator at 6 months [HR 0.97; 95\% confidence interval (CI) 0.73-1.28; $p=0.81$ ] [9] and thus results do not justify extension of MRA use at present.

Patients receiving some cancer chemotherapy treatments may be at risk of developing HF. It is unclear if this risk can be reduced by use of cardioprotective drugs. The Prevention of Cardiac Dysfunction During Adjuvant Cancer Therapy (PRADA) trial (ClinicalTrials.gov \#NCT01434134) randomized 126 breast cancer patients with a baseline $\mathrm{EF}$ of $>50 \%$ being treated with anthracycline \pm trastuzumab and radiation to a protective strategy of candesartan or metoprolol vs. placebo [10]. Patients receiving candesartan had a significantly smaller decline in EF from baseline vs. placebo (0.8\% vs. $2.6 \%$; $p=0.026)$ [10]. No significant benefit was demonstrated for metoprolol [10]. Although small in size, this study suggests potential benefit from candesartan therapy in this patient group.

Previous studies have yielded conflicting conclusions regarding the mortality effect of digoxin among patients with congestive heart failure (CHF) [11, 12]. Madelaire et al. thus conducted an observational study using a Danish nationwide registry of patients discharged from hospital with both $\mathrm{HF}$ and atrial fibrillation (AF), excluding those receiving antiarrhythmic therapy (e.g., amiodarone), those who died within 30 days of discharge, and those not taking a vitamin-K antagonist, then matching the 8078 digoxin-treated patients with 8078 control patients, based on age, sex, comorbidities, CHA2DS2VASc score, medication, and heart-failure severity [13]. The risk of death was reduced [hazard ratio (HR) 0.92; (CI 95\% 0.86-0.98) and risk of readmission increased $\mathrm{HR}$ 1.07; 95\% CI 1.01-1.12] with digoxin users [13]. When death was considered as a competing risk, the collective incidences of readmission were similar at $64.9 \%$ in digoxin users and $64.4 \%$ in non-users [13]. The authors concluded that in digoxin-naive patients with $\mathrm{AF}$, digoxin use was associated with a slightly reduced risk of death [13].

A novel heart failure strategy may be attenuation of myocyte sodium overload by beta 3 agonists. In the Beta 3 Agonists Treatment in HF (BEAT-HF) trial (ClinicalTrials.gov \#NCT01876433) [14, 15], 70 patients with HF were randomized to the beta 3 agonist Mirabegron or placebo for 6 months $[14,15]$. While Mirabegron was not associated with improvement in patients with a baseline $\mathrm{EF}$ $>40 \%$ (mean difference $0.4 \%$; $95 \%$ CI -3.5 to $3.8 ; p=0.82$ ) an exploratory analysis of patients with a baseline $\mathrm{EF} \leq 40 \%$ noted a $5.5 \%$ increase in EF (95\% CI 0.6-10.4; $p<0.03)$ [14, 15]. Further studies are thus planned in patients with severe heart failure [15].

Since some diabetes therapies including some thiazolidinediones and some DPP-4 inhibitors have been associated with an increased risk of HF events, new diabetes therapies require rigorous evaluation of $\mathrm{CV}$ safety. TECOS (Trial Evaluating Cardiovascular Outcomes with Sitagliptin) (ClinicalTrials.gov \#NCT00790205) randomized 14,671 patients with diabetics and $\mathrm{CV}$ disease (history of major coronary artery disease, ischemic cerebrovascular disease, or atherosclerotic peripheral arterial disease) to sitagliptin vs. placebo [16]. At 3 years, there was no difference in the primary endpoint of $\mathrm{CV}$ death, non-fatal MI, non-fatal stroke, or hospitalization for unstable angina for with sitagliptin vs. placebo $(11.4 \%$ vs. $11.6 \%$; $p<0.001$ for noninferiority) [16], no difference 
in total HF hospitalization events (345 vs. 347 placebo; $p=0.996)[16,17]$ and in the subgroup of patients with HF at baseline, no significant difference in hospitalization for $\mathrm{HF}$ (7.4\% vs. 7.0\% HR 1.03; 95\% CI 0.77-1.36; $p=0.86$ ) [17]. Thus, in contrast to some previous gliptin data, no signal to increased HF was seen, which could reflect differences in clinical or patient factors, or a potential intrinsic pharmacologic difference among the DPP-4 inhibitors $[18,19]$.

The sodium glucose cotransporter 2 (SGLT2) is responsible for $90 \%$ of reabsorption of urinary glucose. Inhibition of SGLT2 has been found to significantly reduce $\mathrm{HbA1c}$, weight, and blood pressure and may augment natriuresis [20, 21]. EMPA-REG OUTCOME (ClinicalTrials.gov \#NCT01131676) randomized 7020 patients with T2DM and at high CV risk to the highly selective SGLT2 inhibitor empagliflozin (10 or $25 \mathrm{mg}$ ) or placebo [20, 21]. Patients with eGFR $<30 \mathrm{ml} / \mathrm{min} / 1.73 \mathrm{~m}^{2}$ were excluded. HF was not an exclusion although most patients did not have HF at baseline [20, 21]. Empagliflozin was associated with a $14 \%$ reduction in the primary composite outcome of $\mathrm{CV}$ death, nonfatal MI, or nonfatal stroke $(10.5 \%$ vs. 12.1\%; HR 0.86; 95\% CI 0.74-0.99; $p=0.0382$ for superiority) largely driven by a $38 \%$ reduction in $\mathrm{CV}$ death $(3.67 \%$ vs. $5.87 \%$; HR $0.62 ; 95 \%$ CI $0.49-0.77 ; p<0.0001) \quad$ [20]. Empagliflozin was also associated with a $34 \%$ reduction in $\mathrm{HF}$ hospitalization or $\mathrm{CV}$ death (HR 0.66; 95\% CI 0.55-0.79; $p<0.0001$ ) and $39 \%$ reduction in hospitalization for, or death from, HF (HR 0.61; 95\% CI 0.47-0.79; $p=0.0002$ ) [20]. Although these findings were consistent in both patients with and without HF at baseline, numerically there were very few events in patients without baseline HF [20].

Multiple non-pharmacological HF strategies were presented during 2015. AUGMENT-HF (ClinicalTrials.gov \#NCT01311791) assessed the clinical impact of an alginate-hydrogel LV epicardial implant placed during open thoracotomy (Fig. 1), designed to serve as a LV scaffold with the aim of reducing adverse remodeling [22]. Of 78 patients with advanced $\mathrm{HF}$ and LEF $\leq 35 \%$ randomized to the alginate implant or placebo, 58 patients completed 1-year follow-up [22]. Peak VO2 was significantly improved in the alginate group (mean increase $2.10 \mathrm{ml} / \mathrm{kg} / \mathrm{min} ; \quad 95 \%$ CI 0.96-3.24; $\quad p<0.001) \quad$ with significantly improved NYHA status and 6-min walk test distance $(p<0.001)$ [22]. While a note of caution is needed given nine $(22.5 \%)$ deaths in the alginate vs. four (10.5\%) deaths in the control group $(p=\mathrm{ns})$, the trial was not powered to assess mortality and further clinical trials are planned for this novel approach [22].

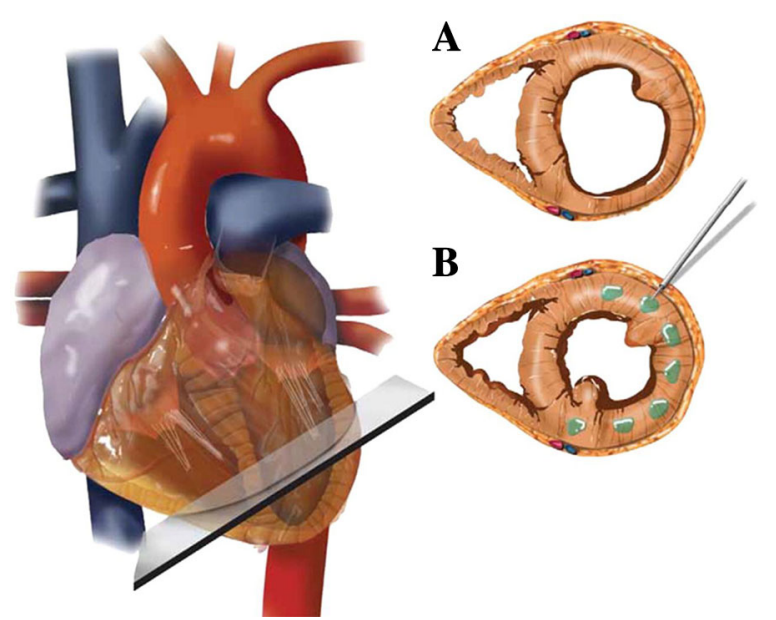

Fig. 1 AUGMENT-HF Alginate-hydrogel LV epicardial implantation at the mid-ventricular level (a) with equal spacing from the antero-septal groove and ending at the postero-septal groove (b). Reproduced with permission from Anker, Coats \& Christian, et al. A prospective comparison of alginatehydrogel with standard medical therapy to determine the impact on functional capacity and clinical outcomes in patients with advanced heart failure (AUGMENTHF trial). Eur Heart J. 2015, 36: 2297-2309 
Large infarcts are associated with degradation of extracellular matrix. Previous preclinical studies and a small human pilot trial have shown that Bioabsorbable Cardiac Matrix (BCM) IK-5001 (an aqueous mixture of sodium alginate and calcium gluconate) when given as an intracoronary bolus can flow to the extracellular space where it forms a flexible hydrogel matrix, thickening the infarct zone, reducing LV wall stress, and potentially reducing adverse remodeling. Once the infarct heals, BCM gradually dissipates and is renally excreted. In PRESERVATION I (ClinicalTrials.gov \#NCT01226563), 303 patients with moderate to large STEMIs (EF $<35 \%$ or MI size $>20 \%$ ) were randomized to intracoronary injection of $4 \mathrm{ml} \mathrm{BCM}(n=201)$ vs. saline control $(n=102) 2-5$ days after their primary PCI [23]. Injection of BCM was not associated with any difference vs. saline in the primary endpoint (change in LV end diastolic volume index (LVEDVI) from baseline to 6 months), or in secondary endpoints including 6-min walk test, NYHA grade, CV hospitalization, or death. BCM injection was associated with five temporary angiographic occlusions vs. one with saline [23]. The disappointing discrepancy between pre-clinical data and PRESERVATION I findings may have been related to microvascular obstruction and edema preventing entry of BCM into the myocardium [23].

HF may be complicated by sleep-disordered breathing and early treatment may improve outcomes. Seventy patients with acute HF diagnosed with sleep apnea by overnight polysomnography within 4 weeks of discharge were treated with continuous positive airway pressure (CPAP) treatment [24]. Of these, 37 (53\%) met the definition of good compliance with the CPAP device [24]. Patients with good CPAP compliance showed a significant decrease in 6-month readmission rates after therapy compared with the 6-month readmission rates pre-therapy (mean $\pm \mathrm{SE}: \quad-1.5 \pm 0.2$ events; $p<0.0001)$ [24].

Better effectiveness after transition-heart failure (BEAT HF) (ClinicalTrials.gov \#NCT01360203) assessed care transition intervention involving pre-discharge education, regular scheduled telephone coaching, and telemonitoring of weight, blood pressure, heart rate, and symptoms in $\mathrm{HF}$ patients [25]. Overall, there was no significant effect on 30- and 180-day readmission $(50.8 \%$ vs. $49.2 \%$, HR 1.03 ; 95\% CI 0.88-1.20; $p=0.74$ ) or 180-day mortality [25]. However, in post hoc analysis, adherence rates with the telephone call and monitoring schedules was noted to be variable. Patients with good telephone call adherence ( $>50 \%$ calls completed) had lower 180-day mortality compared to non-adherent patients $(8.3 \%$ vs. $26 \% ; p \leq 0.001) \quad[25,26]$. Patients with good remote monitoring adherence ( $>50 \%$ days) had lower 180-day mortality $(6.6 \%$ vs. $21.4 \% ; p \leq 0.001)$ and lower 180 -day readmission rates $(41.3 \%$ vs. $61.1 \% ; p \leq 0.001) \quad[25,26]$. While good adherence to a telephone and telemonitoring program appears to be of clinical benefit, as this was a post hoc observation, further prospective investigation is needed.

\section{ANTICOAGULATION FOR ATRIAL FIBRILLATION}

Until recently, the absence of a specific reversal agent for non-vitamin $\mathrm{K}$ antagonist oral anticoagulants (NOACs) has been seen as a limitation. In late 2015, the first specific reversal agent-idarucizumab-was approved for reversal of the direct thrombin inhibitor dabigatran [27]. Approval was based on interim results of REVERSE-AD (ClinicalTrials.gov 
\#NCT02104947) $[28,29]$ in 90 patients-51 with serious bleeding (group A) and 39 requiring an urgent procedure (group B). The anticoagulant effects of dabigatran were rapidly (within minutes) and completely reversed in 88 to $98 \%$ of the patients who had had elevated clotting times at baseline [29]. Unbound dabigatran concentration at $24 \mathrm{~h}$ remained below $20 \mathrm{ng} / \mathrm{ml}$ in $79 \%$ of patients. One thrombotic event occurred within $72 \mathrm{~h}$ of idarucizumab in a patient who had not received anticoagulant re-initiation [29].

Factor Xa oral anticoagulants do not yet have an approved reversal agent but andexanet alfa-a recombinant engineered version of human factor Xa with the ability to bind factor Xa inhibitors-has been tested in healthy older (aged 50-75) volunteers receiving apixaban $5 \mathrm{mg}$ twice daily or rivaroxaban $20 \mathrm{mg}$ once daily [30]. In apixaban participants, andexanet alfa reduced anti-Xa activity by $94 \%$ (vs. 21\% receiving placebo; $p<0.001$ ) and thrombin generation was fully restored in $100 \%$ (vs. $11 \%$ receiving placebo; $p<0.001$ ) within 2 to $5 \mathrm{~min}$ [30]. Among the rivaroxaban participants, andexanet alfa reduced anti-factor Xa activity by $92 \%$ (vs. $18 \%$ receiving placebo) and thrombin generation was fully restored in 96\% (vs. 7\% receiving placebo; $p<0.001$ ) [30]. These effects were sustained when andexanet was administered as a bolus plus an infusion [30]. A phase IIIb/IV trial is now recruiting patients with active bleeding, treated with andexanet.

Balancing antithrombotic efficacy with bleeding safety remains a clinical challenge. REGULATE-PCI (ClinicalTrials.gov \#NCT01848106), randomized patients undergoing PCI to the novel anti IXa anticoagulant pegnivacogin $(n=1616)$ prior to PCI with administration of the specific reversal agent anivamersen at the end of the PCI, or to bivalirudin ( $n=1616)$ (standard bolus, plus infusion for the duration of the PCI) [31]. The trial was halted early as $10 / 1605(0.6 \%)$ patients had severe allergic reactions with the novel regimen (including one fatality) compared to one severe allergic reaction with bivalirudin and had higher rates of bleeding $(p=0.002)$ [31]. However, the primary efficacy outcomes were similar between the two groups, thus suggesting that factor IXa could be a potential target if the allergy issues could be addressed [31].

The Assessment of an Education and Guidance Programme for Eliquis Adherence in Non-Valvular Atrial Fibrillation (AEGEAN) (ClinicalTrials.gov \#NCT01884350) study assessed the impact of an educational program on adherence to apixaban for stroke prevention in $\mathrm{AF}$ [32]. Adherence and persistence were measured by an electronic monitoring device, with non-adherence defined as a 24 -h period where two tablets were missed and non-persistence a 30-day period of discontinuation [32]. Adherence was $88.51 \%$ for standard care and $88.34 \%$ for the educational programme $(p=0.89)$ at week 24 [32]. Persistence at 24 weeks was $90.5 \%$ with standard care and $91.1 \%$ with an educational programme $(p=0.7) \quad[32]$. The authors concluded there was no additional value found so far with an educational programme in the first 6 months of treatment. Long-term adherence will be evaluated in a follow-up study [32].

\section{STRUCTURAL INTERVENTION}

Currently, transcatheter aortic valve replacement (TAVR) is indicated principally in patients at high surgical risk. In the Nordic Aortic Valve Intervention Trial (NOTION) (ClinicalTrials.gov \#NCT01057173), 280 
patients with severe aortic stenosis, an age over 70 but otherwise deemed low risk by the STS (Society of Thoracic Surgeons) score (81\% with a score $<4)$, and deemed suitable for either procedure at a multidisciplinary conference, were randomized to TAVR (Corevalve Medtronic $n=145$ ) or surgical aortic valve replacement (SAVR $n=135$ ) [33]. TAVR compared with SAVR was associated with a similar incidence in the primary endpoint of composite death, stroke, or MI $(13.1 \%$ vs. $16.3 \%, p=0.43$.), less major bleeding $(11.3 \%$ vs. $20.9 \% p=0.03)$, less cardiogenic shock $(10.4 \%$ vs. $4.2 \%(p=0.05)$, less acute kidney injury $(0.7 \% \quad$ vs. $6.7 \% \quad p=0.01)$ and less new/worsening AF at 1 year $(21.2 \%$ vs. $59.4 \%$; $p<0.001)$ although a higher incidence of pacemaker requirement $(38 \%$ vs. $2.4 \%$; $p<0.001)$ and higher incidence of moderate to severe aortic regurgitation at 1 year $(15.7 \%$ vs. 0.9\%; $p<0.001$ ) [33]. Echocardiography rather than computed tomography (CT) was routinely used for pre-procedure measurements, and low TAVR placement was felt to contribute to conduction abnormalities [33]. Although underpowered, NOTION suggests TAVR may be a reasonable alternative to surgical intervention even in low-risk patients [33].

Neurological defects can be an important complication of TAVR. DEFLECT 3 (ClinicalTrials.gov \#NCT02070731) was a small $(n=85)$ randomized trial investigating the use of the TriGuard embolic deflecting device vs. control in patients undergoing TAVR [34]. Complete three-vessel cerebral coverage was achieved in $88.9 \%$ of patients assigned to TriGuard [34]. No significant difference in MACCE (major adverse cardiac or cerebrovascular events) defined as death, stroke, life-threatening or disabling bleeding, acute kidney injury, or major vascular complications was noted (TriGuard 21.7 vs. control 30.8\% subjects $p=0.34$ ) [34]. However, patients with full three-vessel coverage had greater freedom from new brain lesions on magnetic resonance imaging (3.1\% vs. $15.4 \%$ ) (Fig. 2), fewer new neurological deficits (National Institutes of Health Stroke Scale $3.1 \%$ vs. $15.4 \%$ ), and a greater than twofold increase in cognitive recovery at 30 days (assessed by Montreal Cognitive Assessment scores) [34].

The PARTNER II (Placement of AoRTic TraNscathetER Valves) trial (ClinicalTrials.gov \#NCT01314313) evaluated use of valve-in-valve TAVR using the Edwards SAPIEN XT among 197 patients with severe aortic stenosis or regurgitation, a previous surgical bioprosthetic aortic valve and high operative risk [35]. Patients had to be suitable for $23-\mathrm{mm}$ or $26-\mathrm{mm}$ valve and patients with surgical valves $<21 \mathrm{~mm}$ were excluded [35]. At 1 year, the primary endpoint of all-cause mortality was $13.4 \%$, cardiac mortality was $8.9 \%$, stroke rate was $3.7 \%$, and rehospitalization rate was $11.8 \%$ [35]. Peri-procedural major bleeding occurred in $14.8 \%$ and acute kidney injury in $8.2 \%$ [35]. Significant clinical benefit compared with baseline was noted for NYHA status, 6-min walk test, and quality of life [35]. Although non-randomized and sizing issues restrict the applicable population, PARTNER II suggests SAPIEN $\mathrm{XT}$ is a potential alternative to CoreValve for valve-in-valve procedures [35].

In contrast to aortic valve disease, conventional surgery remains the primary treatment for mitral regurgitation in most patients. The optimal surgical approach can be hotly debated. Two-year results of a randomized trial (ClinicalTrials.gov \#NCT00807040) comparing surgical mitral-valve repair with surgical mitral-valve replacement in 251 patients with severe ischemic mitral regurgitation were presented at AHA 2015 [36]. 

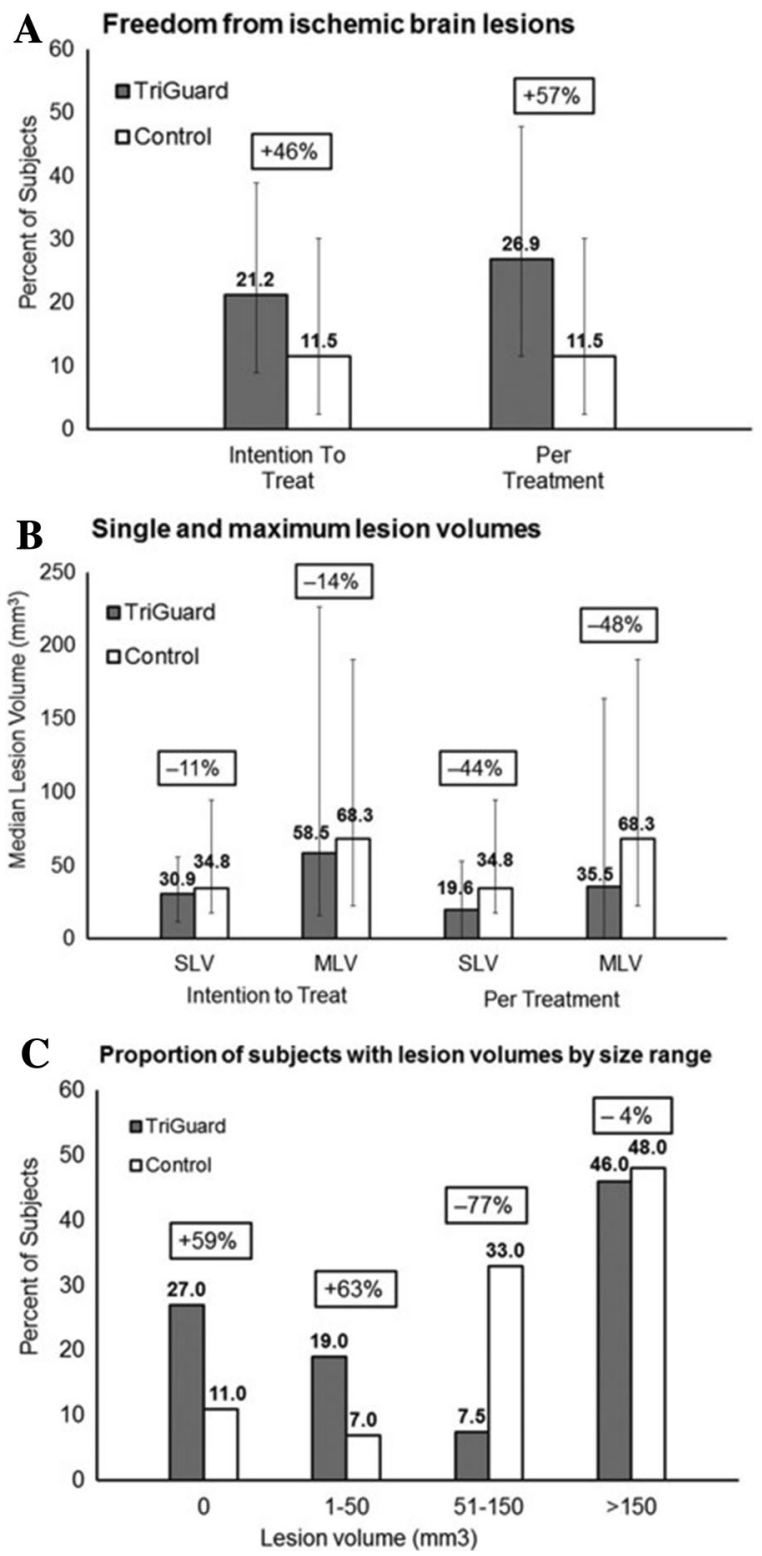

Fig. 2 DEFLECT III (a)\% patients with complete freedom from ischemic brain lesions in the intention-to-treat and per-treatment analysis populations. Error bars represent $95 \%$ confidence intervals. b Per-subject median single and maximum lesion volumes in the intention-to-treat and per-treatment analysis populations. Error bars represent the interquartile range. c Proportion of subjects experiencing a total lesion volume by diffusion-weighted magnetic resonance imaging in the given size range. Reproduced with permission from Lansky, Schofer and Tchetche, et al. A prospective randomized evaluation of the TriGuard $\mathrm{HDH}$ embolic DEFLECTion device during transcatheter aortic valve implantation: results from the DEFLECT III trial. Eur Heart J. 2015; 36: 2070-2078
There was no significant difference in mean left ventricular end-systolic volume index at 2 years ( $52.6 \mathrm{vs.} 60.6 \mathrm{ml} / \mathrm{m}^{2}$ ) for repair vs. replacement, and no difference in 2-year mortality (19.0\% vs. 23.2\% HR 0.79; 95\% CI 0.46-1.35; $p=0.39$ ) [36]. However, repair was associated with a higher rate of moderate or severe mitral regurgitation $(58.8 \%$ vs. $3.8 \% ; p<0.001)$, serious adverse events related to HF $(p=0.05)$ and $\mathrm{CV}$ readmissions $(p=0.01)$ [36].

Ten-year data from the RESPECT trial, investigating the Amplatzer PFO (patent foramen ovale) (St Jude Medical) (ClinicalTrials.gov \#NCT00465270) in preventing recurrent stroke in patients with previous cryptogenic ischemic stroke $(n=499)$ vs. guideline-directed therapy $(n=481)$ were presented at TCT 2015 [37]. PFO closure was not associated with significant reduction in all-cause strokes in the overall intention-to-treat analysis (18 vs. $24 ; p=0.16$ ) but was associated with a significant reduction in cryptogenic stroke (10 vs. 19; HR 0.46; $p=0.042)$ [37]. In patients $<60$ years, PFO closure was associated with a $52 \%$ reduction in all-cause stroke $(p=0.035)$ [37] and in those with atrial septal aneurysmal or substantial shunts, PFO closure was associated with a 75\% reduction in all-cause stroke $(p=0.007)$ [37]. There was no intra-procedure stroke, no device embolization, no device thrombosis, no device erosion, a very low rate of major vascular complications (0.9\%) and device explants (0.4\%) [37]. RESPECT thus supports PFO closure in selected patients.

\section{ATHEROSCLEROSIS}

Despite use of high-dose statin and ezetimibe, patients with elevated LDL cholesterol can remain at elevated $\mathrm{CV}$ risk. Proprotein convertase subtilisin-kexin type 9 (PCSK9) 
reduces LDL-receptor recycling, resulting in a decrease of LDL-C receptors on the surface of hepatocytes and an increase of plasma LDL-C. Inhibition of PCSK9 leads to an increase in surface LDL-C receptors with subsequent lowering of plasma levels of LDL-C (Fig. 3) [38].

In an open-label extension study of OSLER-1 (ClinicalTrials.gov \#NCT01439880) and OSLER-2 (ClinicalTrials.gov \#NCT01854918) patients were randomly assigned in a 2:1 ratio to receive the PCSK9 inhibitor evolocumab (140 mg every 2 weeks or $420 \mathrm{mg}$ monthly) plus standard therapy $(n=2976)$ or standard therapy alone $(n=1487)$ [39]. At 1 year, evolucamab reduced LDL by $61 \%$ from a median $\quad 120-48 \mathrm{mg} / \mathrm{dl} ; \quad p<0.001) \quad$ [39]. Evolucamab was not associated with an increase in serious adverse events $(7.5 \%$ vs. $7.5 \%)$ although neurocognitive side effects were more frequent (0.9\% vs. 0.3\%) [39]. Exploratory analysis suggested evolucamab was associated with a reduction in the endpoint of combined CV events (0.95\% vs. 2.18\%; HR 0.47; 95\% CI $0.28-0.78 ; p=0.003$ ) which will be further assessed in the FOURIER (Further Cardiovascular Outcomes Research With PCSK9 Inhibition in Subjects With Elevated Risk) study (ClinicalTrials.gov \#NCT01764633) [39].

A second PCSK9 inhibitor, alirocumab, was evaluated in the ODYSSEY LONG TERM study (ClinicalTrials.gov number \#NCT01507831) in which 2341 patients with LDL cholesterol $>1.8 \mathrm{mmol} / \mathrm{l}$ despite maximum tolerated statin were randomized in $2: 1$ ratio to alirocumab (150 mg) or placebo subcutaneous injection every 2 weeks for 78 weeks [40]. At week 24 , LDL-C in the alirocumab group had dropped $62 \%$ more from baseline than with placebo $(p<0.001)$; an effect that remained consistent over 78 weeks [40]. Patients receiving alirocumab reported higher rates of myalgia (5.4\% vs. $2.9 \%$ ), neurocognitive events $(1.2 \%$ vs. $0.5 \%)$, and ophthalmologic events (2.9\% vs. $1.9 \%)$ [40]. In exploratory post hoc analysis, MACE events (CHD death, MI, ischemic stroke, or unstable angina requiring hospitalization) were $48 \%$ lower with alirocumab ( $1.7 \%$ vs. 3.3\%; HR 0.52; 95\% CI $0.31-0.90$; nominal $p=0.02$ ), which will be further assessed in the ongoing 18,000-patient, 5-year ODYSSEY OUTCOMES study (ClinicalTrials.gov number \#NCT01663402) [40].

Less than one-third of smokers hospitalized with an acute coronary syndrome remain abstinent following discharge, but the safety of varenicline in this setting remained to be established. The Evaluation of Varenicline in Smoking Cessation for Patients Post-Acute Coronary Syndrome (EVITA) study (ClinicalTrials.gov \#NCT00794573) randomized smokers ( $\geq 10$ cigarettes per day) with acute coronary syndrome (ACS) to varenicline or placebo [41]. The primary endpoint (self-reported cessation at 24 weeks confirmed by carbon monoxide testing) was achieved in $47.3 \%$ of those receiving varenicline vs. $32.5 \%$ receiving placebo $(p=0.012$; number needed to treat 6.8) [41]. At follow-up, continuous abstinence was reported in $35.8 \%$ vs. $25.8 \%(p=0.081)$ and rates of $\geq 50 \%$ reduction in cigarettes were $67.4 \%$ vs. $55.6 \%(p=0.05)$ [41]. There was no significant difference in adverse events except for a higher incidence of abnormal dreams with varenicline (15.2\% vs. $4.6 \%$ ) [41].

Blood pressure targets were relaxed in most recent guidelines [42]. The Systolic Blood Pressure Intervention Trial (SPRINT) (ClinicalTrials.gov \#NCT01206062) randomized 9361 patients aged over 50 with systolic blood pressure $\geq 130 \mathrm{mmHg}$, increased $\mathrm{CV}$ risk, but without diabetes, to a target systolic 



Fig. 3 Catabolism of the LDL particle; the role of PCSK9 and antibody to PCSK9. a Intracellular cholesterol homeostasis is modulated by the LDLR pathway. The LDLR is a cell-surface glycoprotein that is synthesized in the ER and processed in the Golgi apparatus, and transported to the cell surface. The LDLR specifically binds $A p o B$ in LDL particles. The resulting receptor-ligand complex is then internalized by endocytosis, which involves the LDLRAP1. PCSK9 binds the LDLR-LDL complex extracellularly and prevents it from dissociating within the vesicle, thus targeting the whole complex for degradation in the lysosomal compartment. $\mathbf{b}$ Antibody to PCSK9 prevents the binding of PCSK9 to the LDLR-LDL complex. The acidic environment of the internalized vesicle results in the dissociation of the complex. The receptor is recycled to the cell surface, whereas the LDL particle is degraded in the lysosomal compartment. Both LDLR and PCSK9 are transcriptionally regulated by SREBP. Accumulation of free cholesterol released by hydrolysis of cholesteryl esters in the core of LDL inactivates SREBP. LDL, low-density lipoprotein; ApoB, apolipoprotein B; ER, endoplasmic reticulum; LDLR, LDL receptor; LDLRAP1, LDL receptor adaptor protein 1; PCSK9, pro-protein convertase subtilisin/kexin type 9; SREBP, sterol regulatory element-binding protein. Reproduced with permission from Hovingh, Davidson and Kastelein, et al. Diagnosis and treatment of familial hypercholesterolemia. Eur Heart J. 2013; 34: 962-971 
pressure $<120 \mathrm{mmHg}$ (intensive treatment) or a target $<140 \mathrm{mmHg}$ (standard treatment) [43]. The population was typical of a hypertensive population with $28 \%$ of patients over 75 years of age and $28 \%$ with CKD [43]. A mean systolic blood pressure of $121.4 \mathrm{mmHg}$ was reached in the intensive group (achieved on average with 2.8 medications) and $136.2 \mathrm{mmHg}$ (1.8 medications) and standard groups, respectively [43]. The study was stopped early at 3.26 years at which point intensive treatment associated with a $25 \%$ reduction in the primary endpoint of MI, ACS, stroke, heart failure, or death from CV causes (1.65\%/year vs. $2.19 \% /$ year; HR 0.75 ; $95 \%$ CI $0.64-0.89 ; p<0.001)$ and a $27 \%$ reduction in all-cause mortality (3.3\% vs. $4.5 \%$; HR $0.73 ; 95 \%$ CI $0.60-0.90 ; p=0.003$ ) [43]. There was a higher rate of hypotension $(2.4 \%$ vs. $1.4 \%$; HR 1.67; $p=0.001)$, syncope, electrolyte abnormalities, and acute kidney injury/failure, but not of falls leading to injury [43]. The SPRINT results are likely to impact on subsequent hypertension guidelines.

Diuretics remain important treatment options in blood pressure guidelines although they may have adverse effects on blood glucose and potassium. The Prevention And Treatment of Hypertension With Algorithm based therapy 3 (PATHWAY 3) (ClinicalTrials.gov \#NCT00797862) randomized 441 patients with systolic blood pressure $>140 \mathrm{mmHg}$, eligible for diuretic use and with $\geq 1$ other component of metabolic syndrome to therapy with amiloride alone, to combination amiloride plus hydrochlorothiazide, or to hydrochlorothiazide alone [44]. Two-hour glucose concentrations after an oral glucose tolerance test, averaged at 12 and 24 weeks (the primary endpoint), were significantly lower with amiloride alone than hydrochlorothiazide alone $(-0.55 \mathrm{mmol} / \mathrm{l}, 95 \% \mathrm{CI}-0.96$ to -0.14 ; $p=0.0093)$ and in the combination group compared to hydrochlorothiazide alone [ $-0.42 \mathrm{mmol} / \mathrm{l}$ ( -0.84 to -0.004$) ; p=0.048$ ] [44]. Mean home systolic blood pressure was reduced similarly with single treatments, but combination therapy led to an additional $3.4 \mathrm{mmHg}$ reduction compared with hydrochlorothiazide $\quad(95 \% \quad$ CI $\quad 0.9-5.8$; $p=0.007)$ [44]. Hyperkalemia occurred in seven $(4.8 \%)$ amiloride patients compared to three $(2.3 \%)$ in the combination group [44]. In summary, combination therapy appeared to be a favorable treatment option being neutral for glucose and potassium but with greater effects on blood pressure [44].

Lower fasting glucose and HbA1c levels with ranolazine have been suggested by previous trials in the setting of coronary disease $[45,46]$. A prospective randomized study presented at the American Diabetes Association (ADA) 2015 scientific sessions (ClinicalTrials.gov \#NCT01472185) evaluated the effect of ranolazine on type 2 diabetics with a HbA1c of $7-10 \% \quad(53-86 \mathrm{mmol} / \mathrm{mol})$ compared with placebo [47]. Ranolazine compared with placebo was associated with a greater reduction in the primary end point of fall in $\mathrm{HbA1c}$ from baseline at 24 weeks [mean difference $\quad-0.56 \% \quad(-6.1 \mathrm{mmol} / \mathrm{mol})$; $p<0.0001]$ and a greater number of patients achieved HbA1c $<7.0 \%$ at week $24(41.2 \%$ vs. $25.6 \% ; p=0.0004$ ) [47]. Thus, ranolazine may be of particular benefit in patients with coronary disease and diabetes.

\section{BIOMARKERS IN ACS}

The Biomarkers in Acute Cardiovascular Care (BACC) study (ClinicalTrials.gov \#NCT02355457) investigated the different cut-off values for high-sensitivity troponin I and testing at 1 vs. $3 \mathrm{~h}$ for early detection of 
acute MI among 1045 patients presenting with acute ischemic-type chest pain of whom 184 were ultimately diagnosed with acute MI [48]. A cut-off of $6 \mathrm{ng} / \mathrm{l}$ was found to be optimal rather than a conventional 99th percentile value for the assay (27 ng/l) [48]. For rule-out MI, using the $6 \mathrm{ng} / \mathrm{l}$ cut off, there was no difference in negative predictive values between the $1-\mathrm{h}$ [NPV 99.7 95\% CI (98.6-100.0)] and 3-h measurements [negative predictive value (NPV) 100.0 95\% CI (98.5-100.0)] [48]. For rule in MI (defined as levels of $6 \mathrm{ng} / \mathrm{l}$ rising to at least $12 \mathrm{ng} / \mathrm{l})$ there was no significant difference in positive predictive at 1 or $3 \mathrm{~h}[82.895 \% \mathrm{CI}$ (73.2-90.0) vs. 78.6 95\% CI (69.8-85.8)] [48]. The BACC findings are in keeping with other studies suggesting that an accelerated high-sensitivity troponin protocol may enable earlier rule in/rule out of acute MI. Use of additional early biomarkers may improve diagnosis further at this early time point and are under investigation.

\section{ANTIPLATELET THERAPY}

The optimal duration of dual antiplatelet therapy (DAPT) after stenting has been widely debated. The Dual AntiPlatelet Therapy (DAPT) study (ClinicalTrials.gov \#NCT00977938) randomized 11,648 subjects who were able to tolerate initial 12 months of DAPT to an extended further 18 months of DAPT (total 30 months) vs. DAPT discontinuation (total 12 months) [49]. A comparison of subgroups with and without MI was presented at the ACC 2015 scientific sessions. Extended DAPT was associated with reduced in-stent thrombosis (primary endpoint) in those with $\mathrm{MI}(0.5 \%$ vs. $1.9 \% ; p<0.001)$ and without MI (0.4\% vs. $1.1 \%$, $p<0.001$ ) (interaction $p=0.69$ ), reduced new
MI in both post MI and non-MI subgroups, and reduced MACCE (co-primary endpoint) in those with MI (3.9\% vs. $6.8 \% ; p<0.001)$ although only a trend to reduced MACCE in those without MI $(4.4 \%$ vs. $5.3 \% ; p=0.08$; interaction $p=0.03$ ) [49]. However, extended DAPT was associated with increased moderate to severe GUSTO bleeding in both MI (1.9\% vs. $0.8 \% ; p=0.005)$ and non-MI $(2.6 \%$ vs. $1.7 \%$; $p=0.007$ ) patients (interaction $p=0.21$ ) [49].

Long-term DAPT was also explored in PEGASUS-TIMI 54 trial (Prevention of Cardiovascular Events in Patients with Prior Heart Attack Using Ticagrelor Compared to Placebo on a Background of Aspirin-Thrombolysis in Myocardial Infarction 54) (ClinicalTrials.gov \#NCT01225562) [50]. PEGASUS randomized 21,162 patients with a history of MI during the previous 1-3 years to extended standard dose ticagrelor (90 $\mathrm{mg}$ bd), extended low dose (60 mg bd) ticagrelor, or placebo [50].

At a median of 33 months, both ticagrelor groups showed a significant reduction in the incidence of the primary endpoint (CV death, MI or stroke) compared with placebo (7.85\% standard dose ticagrelor (HR 0.85; 95\% CI $0.74-0.96 ; \quad p=0.008) ; \quad 7.77 \%$ reduced dose ticagrelor (HR $0.84 ; \quad 95 \% \quad$ CI $\quad 0.74-0.95$; $p=0.004)$; vs. $9.04 \%$ placebo) [50]. TIMI major bleeding rates were significantly $(p<0.001)$ increased in both ticagrelor groups compared with placebo $(2.60 \%$ standard dose ticagrelor, $2.30 \%$ reduced dose ticagrelor vs. $1.06 \%$ placebo) although the incidence of intracranial hemorrhage or fatal bleeding was similar in all three groups [50]. Dyspnea was significantly more frequent with ticagrelor $(18.93 \%$ standard dose $15.84 \%$ low dose vs. $6.38 \%$ placebo). Standard dose compared with low dose ticagrelor led to numerically higher rates of discontinuation [50]. 
The DAPT and PEGASUS trials suggest that patients at high ischemic risk but low bleeding risk are most likely to benefit from extended therapy A 'DAPT score' has been developed from the DAPT study dataset to help identify patients who may derive net benefit and this, or a similar strategy, is likely to be addressed in future ACS guidelines.

\section{PERCUTANEOUS CORONARY INTERVENTION}

The requirement for at least 3-6 months of DAPT has limited use of drug eluting stents (DES) in patients at high baseline risk of bleeding. The BioFreedom stainless-steel stent incorporates a novel microporous abluminal surface (Fig. 4) that enables controlled release of Biolimus A9, with therapeutic concentrations in local tissue sustained up to 28 days post-implantation [51]. Previous angiographic studies have reported similar late loss to conventional DES while the absence of surface polymer may facilitate early stent healing and a shorter course of DAPT [51]. The LEADERS-FREE trial (ClinicalTrials.gov \#NCT01623180) randomized 2466 patients at high baseline bleeding risk undergoing PCI to BioFreedom or a bare metal stent with DAPT discontinuation after 1 month [52]. At 390 days, BioFreedom was associated with a 50\% reduction in the primary efficacy endpoint of clinically driven TLR (5.1\% vs. 9.8\%; HR 0.50; 95\% CI 0.37-0.69; $p<0.001)$, a $29 \%$ reduction in the primary safety endpoint of $\mathrm{CV}$ death, stent thrombosis or MI $(9.4 \%$ vs. $12.9 \%$; HR $0.71 ; 95 \%$ CI $0.56-0.91 ; p=0.005$ ) (Fig. 5), and a $32 \%$ reduction in secondary endpoint of $\mathrm{MI}(6.1 \%$ vs. $8.9 \%$; HR 0.68 ; 95\% CI $0.50-0.91 ; p=0.01)$ [52]. Biofreedom appears a logical stent choice (rather than bare metal stent or conventional



Fig. 4 BioFreedom stainless-steel stent that incorporates a novel microporous abluminal surface. Reproduced with permission from Biosensors

DES) for patients requiring short-course DAPT or at increased risk of bleeding requiring early DAPT discontinuation.

The role of PCI vs. CABG for treatment of left main coronary disease is the ongoing focus of several large studies. The Premier of Randomized Comparison of Bypass Surgery vs. Angioplasty Using Sirolimus-Eluting Stent in Patients With Left Main Coronary Artery Disease (PreCOMBAT) (ClinicalTrials.gov \#NCT00422968) trial examined patients with left main coronary stenosis receiving PCI $(n=300)$ compared to CABG $(n=300)$ [53]. At 5 years, there was no difference in MACCE (17.5\% PCI vs. 14.3\% CABG; HR 1.27; 95\% CI $0.84-1.90 ; p=0.26)$ or the composite of death, MI or stroke (8.4\% PCI vs. 9.6\% CABG; HR 0.89; 95\% CI $0.52-1.52 ; p=0.66$ ) [53]. Of note, there was higher rate of ischemia-driven target vessel revascularization (TVR) with PCI (11.4\% PCI vs. 5.5\% CABG; HR 2.11; 95\% CI 1.16-3.84; $p=0.012$ ) and 2 PCI patients developed stent thrombosis [53]. However, PreCOMBAT used 


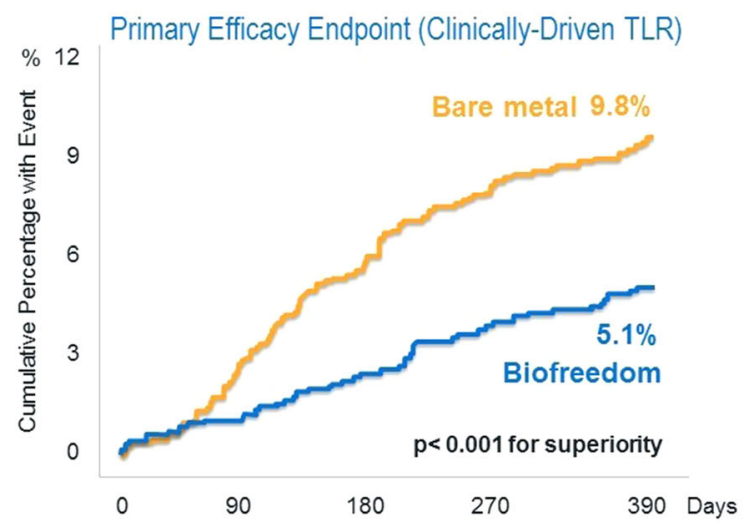

Fig. 5 Primary efficacy and safety endpoints from the LEADERS-FREE trial for the Biofreedom drug-coated stent vs. bare metal stenting. TLR target lesion

first-generation DES, which are associated with higher rates of TVR and late stent thrombosis than latest-generation stents [54]. Results of NOBLE (Nordic-Baltic-British Left Main Revascularization Study) (ClinicalTrials.gov \#NCT01496651) comparing Biomatrix flex with CABG and EXCEL (Evaluation of XIENCE Everolimus Eluting Stent vs. Coronary Artery Bypass Surgery for Effectiveness of Left Main Revascularization) (ClinicalTrials.gov \#NCT01205776) are expected in later 2016.

While the Thrombus Aspiration during Percutaneous coronary intervention in Acute myocardial infarction Study (TAPAS) (Current Controlled Trials \#ISRCTN16716833) suggested a benefit for routine thrombectomy in primary PCI, the Thrombus Aspiration in ST-Elevation Myocardial Infarction in Scandinavia Trial (TASTE) (ClinicalTrials.gov \#NCT01093404) failed to demonstrate a reduction in death, MI, or stent thrombosis [3]. Given concerns that TASTE was underpowered to detect smaller event rates [55], the large Trial of Routine Aspiration Thrombectomy with PCI vs. PCI Alone in Patients with STEMI (TOTAL) (ClinicalTrials.gov \#NCT01149044) was undertaken in which patients with STEMI



revascularization, $M I$ myocardial infarction, $S T$ stent thrombosis. Reproduced with permission from Biosensors

undergoing primary PCI were randomized to routine thrombectomy $(n=5372)$ vs. primary PCI alone $(n=5360)$ [56]. While, like in TAPAS, routine thrombectomy was associated with greater ST segment resolution and less distal embolization, this did not translate into clinical benefit [56]. There was no reduction in the primary outcome (CV death, recurrent $\mathrm{MI}$, cardiogenic shock or NYHA class IV HF at 180 days) (6.9\% vs. $7.0 \%$ respectively, HR 0.99; 95\% CI $0.85-1.15 ; p=0.86$ ) [56], no reduction in the secondary outcome (primary endpoint plus stent thrombosis or TVR within 180 days) (9.9\% thrombectomy vs. $9.8 \%$ PCI alone, HR $1.00 ; 95 \%$ CI $0.89-1.14 ; p=0.95)$, and no reduction in $\mathrm{CV}$ mortality at 180 days $(3.1 \%$ thrombectomy vs. 3.5\% PCI alone, HR 0.90; 95\% CI 0.73-1.12; $p=0.34$ ) [56]. Although numerically low, stroke rates were increased in the thrombectomy compared to PCI alone at both 30 days $(0.7 \%$ vs. $0.3 \%$ respectively, HR 2.06; 95\% CI $1.13-3.75 ; p=0.02)$ and 180 days (52 (1\%) vs. 25 (0.5\%) respectively HR $2.08 ; 95 \%$ CI $1.29-3.35 ; p=0.02$ ) [56]. Thus routine thrombectomy is no longer recommended in primary PCI, although may have a "bail out" role for selected patients [56]. 
DANAMI-3 PRIMULTI (The Third DANish Study of Optimal Acute Treatment of Patients with ST-segment Elevation Myocardial Infarction PRImary PCI in MULTIvessel Disease) (ClinicalTrials.gov \#NCT01960933) compared complete revascularization (with staged pressure wire-guided PCI of non-culprit lesions during index admission) ( $n=314)$ vs. culprit artery intervention alone $(n=313)$ [57]. The complete revascularization strategy was associated with a $44 \%$ reduction in the primary endpoint of death, reinfarction, or ischemia-driven non-culprit artery revascularization ( $13 \%$ vs. $22 \%$; HR 0.56 ; $95 \%$ CI $0.38-0.83 ; p=0.004)$, driven by a $69 \%$ reduction in repeat revascularization (5 vs. 17\%; HR 0.31; 95\% CI 0.18-0.53; $p<0.001)$ of which $40 \%$ was urgent revascularization [57]. Thus, while complete revascularization should usually be undertaken, since there was no difference in death or reinfarction, DANAMI-3 suggests a conservative approach to non-culprit PCI is reasonable if such PCI appears of high risk [57].

No-reflow following PCI may be associated with adverse cardiac outcomes and reduced myocardial salvage (the final infarct size indexed to the initial area at risk). No reflow, if it occurs, is most often observed following stent deployment. The single Korean center INNOVATION trial (NCT02324348) randomized 102 STEMI patients deemed at higher baseline risk of no-reflow following initial thrombectomy and/or balloon angioplasty to deferred stenting at 3-7 days vs. immediate stenting [58]. Deferred stenting was associated with less no-reflow or slow-reflow ( $6 \%$ vs. $29 \% ; p=0.006$ ), less no reflow ( $2 \%$ vs. $14 \% ; \quad p=0.052), \quad$ less intra-procedural thrombotic events (10\% vs. $33 \% ; p=0.010)$ and increased myocardial salvage at 6 months (19.7\% vs. $14.7 \% ; p=0.027$ ) [58]. The study is too small to be conclusive but a larger European deferred stent study is ongoing with results expected in 2016.

Fully bioresorbable stent scaffolds could offer potential long-term vascular advantages over conventional DES although appropriate patient types and safety are still being evaluated. ABSORB Japan (ClinicalTrials.gov \#NCT01844284) presented at ESC 2015, randomized 400 patients undergoing PCI to the Absorb everolimus-eluting bioresorbable vascular scaffold or the Xience everolimus-eluting permanent cobalt-chromium stent [59]. Absorb was non-inferior to Xience for the primary endpoint of target lesion failure [cardiac death, MI attributable to target vessel, or ischemia-driven target lesion revascularization (TLR)] at 12 months $(4.2 \%$ vs. $3.8 \%$; $p$ non-inferiority $<0.0001)$ [59]. There was no difference in the rate of 12-month device thrombosis (although the study was underpowered for this endpoint), 12-month TLR, or 13-month angiographic late lumen loss [59].

The ABSORB III trial (ClinicalTrials.gov \#NCT01751906) randomized 2008 patients undergoing PCI to one or two de novo lesions to Absorb or Xience (in 2:1 fashion) [60]. Absorb was non-inferior to Xience for the primary endpoint of target lesion failure at 1 year $(7.8 \%$ vs. $6.1 \% ; p$ non-inferiority $=0.007)$ with no difference in individual endpoint components [60]. Of note, Absorb was associated with a higher rate of subacute ( $>24 \mathrm{~h}$ to 30 days) device thrombosis ( $0.9 \%$ vs. 0.1\%; RR 6.26; 95\% CI 0.82-48.04; $p=0.04$ ) although the relative difference was less at 1 year (1.5\% vs. $0.7 \%$; RR $2.08 ; 95 \%$ CI $0.78-5.51 ; \quad p=0.13) \quad$ [60]. Intravascular imaging is usually recommended to guide optimal B vs. deployment but a surprisingly 
low use was noted in ABSORB III. The first-generation Absorb B vs. has relatively thick struts and device thrombosis was less prevalent in vessels $>2.25 \mathrm{~mm}$. Nevertheless, a meta-analysis of six trials including ABSORB Japan and ABSORB III did confirm a small but significant increase in device thrombosis [61]. Thus, appropriate patient/lesion selection, careful lesion preparation, optical coherence tomography to guide sizing \pm post dilatation and extended DAPT (to 2 years) is recommended minimize risk of device thrombosis.

Reducing bleeding complications following PCI is an important goal given the association of bleeding with adverse clinical outcomes. The MATRIX (Minimizing Adverse Haemorrhagic Events By Transradial Access Site And Systemic Implementation Of Angiox) (ClinicalTrials.gov \#NCT01433627) study randomized 8404 patients with ACS, undergoing urgent angiography (and 80\% with follow-on PCI) to radial or femoral access $[62,63]$. Radial access was associated with a significant reduction in non-CABG major bleeding (1.6\% vs. $2.3 \%$, RR $0.67 ; 95 \%$ CI $0.49-0.92 ; p=0.013)$, a $14.6 \%$ trend to reduction in the primary endpoint of MACE (death, MI or stroke; $8.8 \%$ vs. $10.3 \%$; 95\% CI $\quad 0.74-0.99 ; \quad p=0.0307$ although not-significant at an $\alpha$ of 0.025) [62] and significant reductions in secondary endpoints of all-cause mortality ( $1.6 \%$ vs. $2.2 \%$, RR 0.72 ; 95\% CI 0.53-0.99; $p=0.045$ ) and NACE (net composite of death, MI, stroke, or major bleeding; $9.8 \%$ vs. $11.7 \%$; HR $0.83 ; 95 \%$ CI 0.73-0.96; $p=0.0092$ ) [62]. Such results strongly support radial access being preferred as the default access site. A separate randomization within the MATRIX study to bivalirudin vs. unfractionated heparin \pm bail out glycoprotein IIb/IIIa inhibition did not show any difference in MACE or NACE.
In some patients with poor radial access, the ulnar artery route, although technically more challenging, may be a useful alternative to avoid femoral cannulation. The single-center Ajmer Ulnar Artery Working Group Study (AJULAR) presented at ACC2015 randomized 2533 patients undergoing PCI by experienced operators ( $>50$ ulnar procedures) to ulnar or radial access [64]. There was no significant difference in the primary endpoint (composite MACE, site cross-over, major vascular events during hospital stay; $14.6 \%$ TUA vs. $14.4 \%$ TRA $95 \% \mathrm{CI} ; p=0.92$ ), or individual components of the composite endpoint [64].

\section{ARRHYTHMIA AND DEVICES}

Requirement for creation of a subcutaneous pacemaker pocket and insertion of a transvenous lead in conventional pacing systems can be associated with important complications such as pocket hematoma or infection, pneumothorax, or hemothorax. The Micra Transcatheter Pacing System Study (ClinicalTrials.gov \#NCT02004873) evaluated use of a leadless pacemaker (Fig. 6) implanted in the right ventricular apex via femoral vein implantation and secured by small tines in 725 patients (719 successfully implanted) [65]. The primary safety endpoint (freedom from system or procedure related major complications) was achieved in $96 \%$ and the primary efficacy endpoint (percentage of patients with low and stable pacing capture thresholds at 6 months) in $98.3 \%$-both of which were higher than the minimum performance goals from conventional pacing historical control data [65]. Major complications, which were less common than in the historical control dataset, included cardiac perforation or effusion/tamponade (1.6\%), groin site 


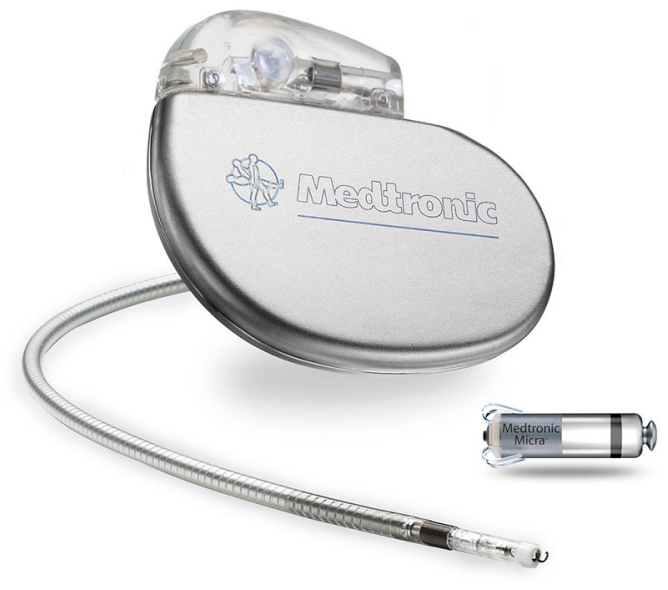

Fig. 6 Traditional pacemaker vs. Micra. Reproduced with permission of Medtronic, Inc

complication $(0.7 \%)$, and pacing issues $(0.3 \%)$ but no (0\%) dislodgments [65].

Another leadless pacemaker-the Nanostim LP (St. Jude Medical)—was evaluated among 300 patients in the LEADLESS II study (ClinicalTrials.gov \#NCT02030418) [66]. The primary safety endpoint (6-month freedom from device-related serious adverse events) was met in $93.3 \%$ and primary efficacy endpoint (acceptable 6-month pacing threshold and sensing amplitude) was met in 90\%-both also exceeding minimum performance goals from conventional pacing historical control data [66]. In total, $6.7 \%$ had serious adverse events (1.7\% device dislodgement with percutaneous retrieval, $1.3 \%$ cardiac perforation and 1.3\% pacing-threshold elevation requiring percutaneous retrieval and device replacement) [66]. As each of the leadless pacemaker studies are observational, larger randomized studies with longer term data including safety and retrieval data is required.

$\mathrm{AF}$ is present in $30-50 \%$ of patients presenting for mitral valve surgery. Current guidelines suggest it is reasonable to perform
AF ablation in selected patients undergoing cardiac surgery but acknowledge that supporting data are limited. Thus, in a Cardiothoracic Surgical Trials Network (CTSN) study (ClinicalTrials.gov \#NCT00903370) 260 patients with persistent including longstanding persistent AF requiring mitral valve surgery were randomized to ablation or no-ablation groups [67]. Patients assigned to ablation were further randomized to pulmonary vein isolation (PVI) or the more complex biatrial maze. Those receiving concomitant $\mathrm{AF}$ ablation were over twice as likely to be free from AF on 72-h Holter at both 6 and 12 months $(63.2 \%$ vs. $29.4 \%$; $p<0.001)$ although there no difference whether performed by PVI or biatrial maze (61\% vs. 66\%; $p=0.6)$ [67]. Ablation was associated with increased need for permanent pacemaker implantation (21.5 vs. 8.1 per 100 patient-years, $p=0.01$ ) [67]. There was no difference in major cardiac or cerebrovascular adverse events at 12 months but the study was not powered to show benefit in this respect.

Although the CTSN study did not find an advantage for the more complex biatrial maze over PVI, with a transcatheter approach, persistent AF may benefit more extensive ablation. The BELIEF trial (ClinicalTrials.gov \#NCT01362738) randomized 173 patients with long-standing persistent AF undergoing pulmonary vein antrum and non-pulmonary vein trigger ablation to additional left atrial appendage (LAA) electrical isolation $(n=85)$ or no LAA isolation $(n=88)$ [68]. Those undergoing LAA electrical isolation had significantly higher freedom from $\mathrm{AF}$ at 1-year (56\% vs. 28\%; HR, 1.92; $p=0.001$ ) [68]. There was no significant difference in secondary endpoints including mortality, stroke, or re-hospitalization [68].

Anticoagulation strategy was assessed in the randomized ActiVe-controlled multi-cENTer 
stUdy with blind-adjudication designed to evaluate the safety of uninterrupted Rivaroxaban and uninterrupted vitamin $\mathrm{K}$ antagonists [VKA] in subjects undergoing cathEter ablation for non-valvular atrial fibrillation (VENTURE-AF; ClinicalTrials.gov \#NCT01729871, $n=248$ patients) [69]. No adverse safety endpoints were reported for rivaroxaban and only rarely for VKA (major bleeding $0.4 \%$; composite of stroke/systemic embolism/MI/vascular death/bleeding $0.8 \%$ ) [69]. While the trial was too small to assess superiority, it suggests rivaroxaban is a reasonable alternative to vitamin $\mathrm{K}$ antagonists in patients undergoing catheter ablation in the setting of uninterrupted anticoagulation [69].

The LEGACY Study (Long-Term Effect of Goal directed weight management on AF cohort: a 5-year follow-up study) (ACTRN12614001123639) evaluated the long-term impact of weight loss and weight fluctuation on rhythm control in obese individuals with symptomatic paroxysmal or persistent AF [70]. All patients received weight loss counseling with optional participation in a weight management clinic or self-managed program [70]. Those achieving $\geq 10 \%$ vs. $<10 \%$ weight loss had reduced AF burden and symptom severity $(p<0.001)$ and a sixfold greater likelihood of arrhythmia-free survival $(p<0.001)$ irrespective of rhythm control strategy [70]. Multivariate analyses found weight loss and weight fluctuation to be independent predictors of outcomes $(p<0.001)$ [70].

Outcomes following out of hospital cardiac arrest remain poor. The Trial of Continuous or Interrupted Chest Compressions during CPR conducted by the Resuscitation Outcomes Consortium) (ClinicalTrials.gov \#NCT01372748) assessed whether outcomes with non-trauma related cardiac arrest after continuous compressions with positive-pressure ventilation (intervention group) differed from those after compressions that were interrupted for ventilations at a ratio of 30 compressions to two ventilations (control group) [71]. However, there was no significant difference in survival to hospital discharge ( $8.9 \%$ vs. $9.7 \%, 95 \%$ CI -1.5 to $0.1 ; p=0.07$ ) or recovery of favorable neurologic function at discharge $(7.0 \%$ vs. $7.7 \%, 95 \%$ CI -1.4 to 0.1 ; $p=0.09)$ [71].

\section{IMAGING}

The Prospective Multicenter Imaging Study for Evaluation of Chest Pain (PROMISE) study (ClinicalTrials.gov \#NCT01174550) randomized 10,003 symptomatic patients to a strategy of initial anatomical testing with the use of coronary computed tomographic angiography (CTA) or to functional testing (nuclear stress testing 68\%, stress echocardiography $22 \%$ or exercise electrocardiography 10\%) [72]. Median cumulative radiation exposure per patient was lower with CTA (driven by significantly higher exposure with nuclear imaging vs. CTA) [72]. Those assigned to CTA vs. functional testing were more likely to undergo cardiac catheterization $(12.2 \%$ vs. $8.1 \%)$ but of these, most patients in the CTA group (72.1\%) had obstructive coronary disease, whereas less than half in the functional group (47.5\%) had obstructive coronary disease) [72]. Revascularization $<90$ days was undertaken in $6.2 \%$ of the CTA group vs. $3.2 \%$ in the functional group $(p<0.001)$, including 72 patients and 38 patients, respectively, who underwent CABG [72]. Over a median follow-up of 25 months there was no difference in the incidence of the primary end-point (death, MI, hospitalization for 
unstable angina, or major procedural complication; $3.3 \%$ vs. $3.0 \%$; adjusted HR 1.04; 95\% CI $0.83-1.29 ; p=0.75$ ) [72]. Thus, CTA appears to identify coronary disease more accurately than functional testing although the clinical impact in PROMISE was unclear.

A second large multicenter CTA study (The SCOT-HEART trial) has helped to define the clinical impact of CTA further. SCOT-HEART randomized 9849 patients with an initial clinical diagnosis of coronary heart disease (CHD) in $47 \%$ and angina due to $\mathrm{CHD}$ in $36 \%$ to usual care plus CTA at $\sim 6$ weeks or to usual care alone (ClinicalTrials.gov \#NCT01149590) [73]. Use of CTA reclassified diagnosis of CHD in $27 \%$ and angina in $23 \%$ (vs. $1 \%$ and $1 \%$, respectively, with usual care $p<0.0001$ ) and led to a change in planned investigation in 15\% (cancellation of 121 functional tests, cancellation of 29 invasive angiograms, but scheduling of an additional 94 invasive angiograms, $88 \%$ of which showed obstructive disease requiring revascularization) [73]. At a median follow-up of 1.7 years, there was a $38 \%$ trend to reduction in fatal and non-fatal MI (26 vs. 42, HR 0.62, 95\% CI $0.38-1.01 ; p=0.0527$ ) [73]. SCOT-HEART thus confirmed that CTA helps clarify diagnosis, enables more appropriate use of invasive angiography/intervention and may reduce the future risk of MI [73].

\section{CONCLUSIONS}

In this paper, we have highlighted and summarized numerous important studies in the field of cardiology that have been presented to major international cardiology meetings throughout 2015. Many of these studies will contribute to updating of current practice guidelines and others will play an integral role in the advancement and development of new therapeutic strategies.

\section{ACKNOWLEDGMENTS}

No funding or sponsorship was received for this study or publication of this article. All named authors meet the International Committee of Medical Journal Editors (ICMJE) criteria for authorship for this manuscript, take responsibility for the integrity of the work as a whole, and have given final approval for the version to be published.

Disclosures. Claire McCune and Peter McKavanagh declare they have no conflicts of interest. Ian Menown has received grants to institution, honoraria and/or conference sponsorship from: Boston Scientific, Sanofi Aventis, Biosensors, Meril Life, Orbus Neich, Menarini, Astra Zeneca, Bayer, Boehringer Ingelheim, Daichii Sankyo, Lilly, Bristol Myers Squibb, and Pfizer.

Compliance with Ethics Guidelines. This article is based on previously conducted studies and does not involve any new studies of human or animal subjects performed by any of the authors.

Open Access. This article is distributed under the terms of the Creative Commons Attribution-NonCommercial 4.0 International License (http://creativecommons.org/licenses/ by-nc/4.0/), which permits any noncommercial use, distribution, and reproduction in any medium, provided you give appropriate credit to the original author(s) and the source, provide a link to the Creative Commons license, and indicate if changes were made. 


\section{REFERENCES}

1. Filippatos G, Anker S, Böhm M, Gheorghiade M, Køber L, Krum H, Maggioni A, Ponikowski P, Voors A, Zannad F, Kim S, Nowack C, Palombo G, Kolkhof P, KimmeskampKirschbaum N, Pieper A and Pitt B. Results of ARTS-HF: finerenone versus eplerenone in patients with worsening chronic heart failure and diabetes and/or chronic kidney disease. https://www.escardio.org/static_file/Escardio/Pressmedia/Press\%20releases/2015/Congress/ARTS-HF_ Filippatos.pdf. Accessed 15 Jan 2016.

2. Pitt B, Anker SD, Bohm M, et al. Rationale and design of MinerAlocorticoid Receptor antagonist Tolerability Study-Heart Failure (ARTS-HF): a randomized study of finerenone vs. eplerenone in patients who have worsening chronic heart failure with diabetes and/or chronic kidney disease. Eur J Heart Fail. 2015;17(2):224-32.

3. McKavanagh P, McCune C, Menown IB. A review of the key clinical trials of 2014. Cardiol Ther. 2015;4(1):5-23.

4. Weir MR, Bakris GL, Bushinsky DA, et al. Patiromer in patients with kidney disease and hyperkalemia receiving RAAS inhibitors. $\mathrm{N}$ Engl $\mathrm{J}$ Med. 2015;372(3):211-21.

5. Pitt B, Bakris G, Weir M, et al. One-year safety and efficacy of patiromer for hyperkalemia in heart failure patients with chronic kidney disease on renin-angiotensin-aldosterone system inhibitors: results from AMETHYST-DN. American College of Cardiology 2015 Scientific Sessions, San Diego (2015).

6. McMurray JJ, Packer M, Desai AS, et al. Angiotensin-neprilysin inhibition versus enalapril in heart failure. $\mathrm{N}$ Engl J Med. 2014;371(11):993-1004.

7. Damman K, Andersen K, Belohlavek J, et al. Angiotensin receptor neprilysin inhibition and renal function in heart failure: results from PARADIGM-HF. European Society of Cardiology 2015 Congress, London (2015).

8. Zannad F, McMurray JJ, Krum H, et al. Eplerenone in patients with systolic heart failure and mild symptoms. N Engl J Med. 2011;364(1):11-21.

9. Beygui F, Cayla G, Roule V, et al. Early aldosterone blockade in acute myocardial infarction: The randomized ALBATROSS trial. European Society of Cardiology 2015 Congress. London (2015).

10. Gulati G, Heck SL, Hoffman P, et al. Prevention of cardiac dysfunction during adjuvant breast cancer therapy (PRADA): primary results of a randomized,
2 x 2 factorial, placebo-controlled, double-blind clinical trial American Heart Association 2015 Scientific Sessions. Orlando (2015).

11. Whitbeck MG, Charnigo RJ, Khairy $P$, et al. Increased mortality among patients taking digoxin-analysis from the AFFIRM study. Eur Heart J. 2013;34(20):1481-8.

12. Gheorghiade M, Fonarow GC, van Veldhuisen DJ, et al. Lack of evidence of increased mortality among patients with atrial fibrillation taking digoxin: findings from post hoc propensity-matched analysis of the AFFIRM trial. Eur Heart J. 2013;34(20):1489-97.

13. Madelaire C, Schou M, Kristensen KE, et al. Use of digoxin is safe in patients with atrial fibrillation and heart failure: a nationwide propensity-matched study. European Society of Cardiology 2015 Congress. London (2015).

14. Bundgaard $\mathrm{H}$, Axelsson $\mathrm{A}$, Iversen $\mathrm{K}$, et al. The-first-in-man randomized trial of $a$ $\beta 3$-adrenoceptor agonist in chronic heart failure: the BEAT-HF trial. American Heart Association (AHA) 2015 Scientific Sessions. Orlando (2015).

15. Bundgaard $\mathrm{H}$, Axelsson $\mathrm{A}$, Thomsen $\mathrm{JH}$, et al. The-first-in-man randomized trial of a ß3-adrenoceptor agonist in chronic heart failure. 2015. http://www.clinicaltrialresults.org/Slides/ AHA2015/Bundgaard_BEAT.pptx. Accessed 13 Jan 2016.

16. Green JB, Bethel MA, Armstrong PW, et al. Effect of sitagliptin on cardiovascular outcomes in Type 2 diabetes. N Engl J Med. 2015;373(3):232-42.

17. Armstrong P, Van de Werf F. TECOS Trial Evaluating Cardiovascular Outcomes with Sitagliptin. Impact of Sitagliptin on Heart Failure and Related Outcomes. European Society of Cardiology 2015 Congress. London (2015).

18. Scirica BM, Bhatt DL, Braunwald E, et al. Saxagliptin and cardiovascular outcomes in patients with Type 2 diabetes mellitus. $\mathrm{N}$ Engl J Med. 2013;369(14):1317-26.

19. White WB, Cannon CP, Heller SR, et al. Alogliptin after acute coronary syndrome in patients with type 2 diabetes. N Engl J Med. 2013;369(14):1327-35.

20. Inzucchi SEWC, Lachen JM, et al. Empagliflozin and cardiovascular outcomes in patients with Type 2 diabetes mellitus at high cardiovascular risk. American Heart Association 2015. Orlando (2015).

21. Zinman B, Wanner C, Lachin JM, et al. Empagliflozin, cardiovascular outcomes, and 
mortality in type 2 diabetes. $\mathrm{N}$ Engl J Med. 2015;373(22):2117-28.

22. Mann DL, Lee RJ, Coats AJ, et al. One-year follow-up results from AUGMENT-HF: a multicentre randomized controlled clinical trial of the efficacy of left ventricular augmentation with Algisyl in the treatment of heart failure. Eur J Heart Fail. 2016;18(3):314-25.

23. Zeymer U, Rao SV, Krucoff MW, et al. A placebo-controlled, multicenter, randomized, double blind trial to evaluate the safety and effectiveness of IK-5001 (Bioabsorbable Cardiac Matrix $[\mathrm{BCM}]$ ) for the prevention of remodeling of the ventricle and congestive heart failure after acute myocardial infarction. European Society of Cardiology 2015 Congress, London (2015).

24. Sharma S, Mather P, Gupta A, et al. Effect of early intervention with positive airway pressure therapy for sleep disordered breathing on six-month readmission rates in hospitalized patients with heart failure. Am J Cardiol. 2016;117(6):940-5.

25. Ong MK, Romano PS, Edgington $S$, et al. Effectiveness of remote patient monitoring after discharge of hospitalized patients with heart failure: the better effectiveness after transition-heart failure (BEAT-HF) randomized clinical trial. JAMA Intern Med. 2016;176(3):310-8.

26. Ong MK, Romano PS, Edgington S, et al. Remote patient management after discharge of hospitalized heart failure patients: the better effectiveness after transition-heart failure (BEAT-HF) study. American Heart Association (AHA) 2015 Scientific Sessions. Orlando (2015). http://www. clinicaltrialresults.org/Slides/AHA2015/Ong_Heart FailureStudy.pptx. Accessed 14 Jan 2016.

27. EMC. Praxbind $2.5 \mathrm{~g} / 50 \mathrm{ml}$ solution for injection/ infusion. https://www.medicines.org.uk/emc/ medicine/31243.

28. Levy JH, Verhamme P, Sellke FW, Reilly PA, Dubiel R, Eikelboom J, E Hylek, Kreuzer J, Weitz JL, Pollack C. Initial experience with idarucizumab in dabigatran-treated patients requiring emergency surgery or intervention: interim results from the RE-VERSE AD study. European Society of Cardiology 2015 Congress. London (2015).

29. Pollack CV Jr, Reilly PA, Eikelboom J, et al. Idarucizumab for Dabigatran Reversal. N Engl J Med. 2015;373(6):511-20.

30. Siegal DM, Curnutte JT, Connolly SJ, et al. Andexanet Alfa for the reversal of factor Xa inhibitor activity. N Engl J Med. 2015;373(25):2413-24.
31. Lincoff AM, Mehran R, Povsic TJ, et al. Effect of the REG1 anticoagulation system versus bivalirudin on outcomes after percutaneous coronary intervention (REGULATE-PCI): a randomised clinical trial. Lancet. 2016;387(10016):349-56.

32. Montalescot $G$, Brotons C, Cosyns B, Crijns $H$, D'Angelo A, Drouet L et al. Assessment of an education and guidance program for apixaban adherence in non-valvular atrial fibrillation: the randomised AEGEAN study. European Society of Cardiology 2015 Congress. London (2015).

33. Thyregod HG, Steinbruchel DA, Ihlemann N, et al. Transcatheter Versus surgical aortic valve replacement in patients with severe aortic valve stenosis: 1-year results from the all-comers NOTION randomized clinical trial. J Am Coll Cardiol. 2015;65(20):2184-94.

34. Lansky AJ, Schofer J, Tchetche D, et al. A prospective randomized evaluation of the TriGuard HDH embolic DEFLECTion device during transcatheter aortic valve implantation: results from the DEFLECT III trial. Eur Heart J. 2015;36(31):2070-8.

35. Dvir D, Webb JG, Mack MJ. PARTNER II valve-in-valve registry: evaluation of a balloon-expandable transcatheter aortic valve in patients with failed bioprosthetic surgical aortic valves. Transcatheter cardiovascular therapeutics 2015. San Francisco (2015).

36. Goldstein D, Moskowitz AJ, Gelijns AC, et al. Two-year outcomes of surgical treatment of severe ischemic mitral regurgitation. $\mathrm{N}$ Engl J Med. 2016;374(4):344-53.

37. Carroll JD, MacDonald L, Marks D, Tirschwell D. RESPECT Extended Follow-up Results. Transcatheter Cardiovascular Therapeutics 2015. San Francisco (2015).

38. Hovingh GK, Davidson MH, Kastelein JJ, O'Connor AM. Diagnosis and treatment of familial hypercholesterolaemia. Eur Heart J. 2013;34(13):962-71.

39. Sabatine MS, Giugliano RP, Wiviott SD, et al. Efficacy and safety of evolocumab in reducing lipids and cardiovascular events. $\mathrm{N}$ Engl J Med. 2015;372(16):1500-9.

40. Robinson JG, Farnier M, Krempf M, et al. Efficacy and safety of alirocumab in reducing lipids and cardiovascular events. $\mathrm{N}$ Engl J Med. 2015;372(16):1489-99.

41. Eisenberg MJ, Windle SB, Roy N, et al. Varenicline for smoking cessation in hospitalized patients with acute coronary syndrome. Circulation. 2016;133:21-30. 
42. Mancia G, Fagard R, Narkiewicz K, et al. ESH/ESC Guidelines for the management of arterial hypertension. The Task Force for the management of arterial hypertension of the European Society of Hypertension (ESH) and of the European Society of Cardiology (ESC) 2013;34(28):2159-219.

43. Wright JT, Williamson JD, Whelton PK, The SPRINT Research Group, et al. A randomized trial of intensive versus standard blood-pressure control. N Engl J Med. 2015;373(22):2103-16.

44. Brown MJ, Williams B, Morant SV, et al. Effect of amiloride, or amiloride plus hydrochlorothiazide, versus hydrochlorothiazide on glucose tolerance and blood pressure (PATHWAY-3): a parallel-group, double-blind randomised phase 4 trial. Lancet Diabetes Endocrinol; 2016;4(2):136-47.

45. Kosiborod M, Arnold SV, Spertus JA, et al. Evaluation of ranolazine in patients with type 2 diabetes mellitus and chronic stable angina: results from the TERISA randomized clinical trial (Type 2 Diabetes Evaluation of Ranolazine in Subjects With Chronic Stable Angina). J Am Coll Cardiol. 2013;61(20):2038-45.

46. Morrow DA, Scirica BM, Chaitman BR, et al. Evaluation of the glycometabolic effects of ranolazine in patients with and without diabetes mellitus in the MERLIN-TIMI 36 randomized controlled trial. Circulation. 2009;119(15):2032-9.

47. Eckel RH, Henry RR, Yue P, et al. Effect of ranolazine monotherapy on glycemic control in subjects with type 2 diabetes. Diabetes Care. 2015;38(7):1189-96.

48. Westermann D, Neumann JT, Sörensen NA, et al. Accurate and rapid diagnosis of myocardial infarction using a high-sensitivity troponin I 1-hour algorithm. European Society of Cardiology 2015 Congress. London (2015).

49. Yeh RW, Kereiakes DJ, Steg PG, et al. Benefits and risks of extended duration dual antiplatelet therapy after PCI in patients with and without acute myocardial infarction. J Am Coll Cardiol. 2015;65(20):2211-21.

50. Bonaca MP, Bhatt DL, Cohen M, et al. Long-term use of ticagrelor in patients with prior myocardial infarction. N Engl J Med. 2015;372(19):1791-800.

51. Shand JA, Kodoth V, Menown IBA. Novel stent and drug elution technologies. Interv Cardiol. 2011;3(4):473-81.

52. Urban P, Meredith IT, Abizaid A, et al. Polymer-free drug-coated coronary stents in patients at high bleeding risk. $\mathrm{N}$ Engl J Med. 2015;373(21):2038-47.
53. Ahn JM, Roh JH, Kim YH, et al. Randomized trial of stents versus bypass surgery for left main coronary artery disease: 5-year outcomes of the PRECOMBAT study. J Am Coll Cardiol. 2015;65(20):2198-206.

54. Serruys PW, Farooq V, Kalesan B, et al. Improved safety and reduction in stent thrombosis associated with biodegradable polymer-based biolimus-eluting stents versus durable polymer-based sirolimus-eluting stents in patients with coronary artery disease: final 5-year report of the LEADERS (Limus Eluted From A Durable Versus ERodable Stent Coating) randomized, noninferiority trial. JACC Cardiovasc Interv. 2013;6(8):777-89.

55. Lagerqvist B, Fröbert O, Olivecrona GK, et al. Outcomes 1 year after thrombus aspiration for myocardial infarction. N Engl J Med. 2014;371(12):1111-20.

56. Jolly SS, Cairns JA, Yusuf S, et al. Randomized trial of primary PCI with or without routine manual thrombectomy. N Engl J Med. 2015;372(15):1389-98.

57. Engstrom T, Kelbaek H, Helqvist S, et al. Complete revascularisation versus treatment of the culprit lesion only in patients with ST-segment elevation myocardial infarction and multivessel disease (DANAMI-3-PRIMULTI): an open-label, randomised controlled trial. Lancet. 2015;386(9994):665-71.

58. Yu CW. INNOVATION: a prospective randomized trial of primary versus deferred stent implantation in patients with st-segment elevation myocardial infarction. Transcatheter cardiovascular therapeutics 2015. San Francisco (2015).

59. Kimura T, Kozuma K, Tanabe K, et al. A randomized trial evaluating everolimus-eluting Absorb bioresorbable scaffolds vs. everolimus-eluting metallic stents in patients with coronary artery disease: aBSORB Japan. Eur Heart J. 2015;36(47):3332-42.

60. Ellis SG, Kereiakes DJ, Metzger DC, et al. Everolimus-eluting bioresorbable scaffolds for coronary artery disease. $\mathrm{N}$ Engl J Med. 2015;373(20):1905-15.

61. Stone GW, Gao R, Kimura T, et al. 1-year outcomes with the Absorb bioresorbable scaffold in patients with coronary artery disease: a patient-level, pooled meta-analysis. Lancet. 2016;387(10025):1277-89.

62. Valgimigli M, Gagnor A, Calabró P, et al. Radial versus femoral access in patients with acute coronary syndromes undergoing invasive management: a randomised multicentre trial. Lancet. 2015;385(9986):2465-76.

63. Valgimigli M. MATRIX group. Bivalirudin infusion compared to unfractionated heparin in patients with acute coronary syndromes undergoing 
invasive management: results from the minimizing adverse haemorrhagic events by transradial access site and systemic implementation of angiox (MATRIX) antithrombin program. American College of Cardiology 2015 Scientific Sessions. San Diego (2015).

64. Gokhroo R, Kishor K, Ranwa B, et al. Ulnar artery interventions non-inferior to radial approach: aJmer Ulnar ARtery (AJULAR) Intervention Working Group Study Results. J Invasive Cardiol. 2016;28(1):1-8.

65. Reynolds D, Duray GZ, Omar R, et al. A leadless intracardiac transcatheter pacing system. N Engl J Med. 2016;374(6):533-41.

66. Reddy VY, Exner DV, Cantillon DJ, et al. Percutaneous implantation of an entirely intracardiac leadless pacemaker. $\mathrm{N}$ Engl J Med. 2015;373(12):1125-35.

67. Gillinov AM, Gelijns AC, Parides MK, et al. Surgical ablation of atrial fibrillation during mitral-valve surgery. N Engl J Med. 2015;372(15):1399-409.

68. Di Biase L BJ, Mohanty P, et al. Effect of empirical left atrial appendage isolation on long-term procedure outcome in patients with long-standing persistent atrial fibrillation undergoing catheter ablation: results of the BELIEF randomized trial.
European Society of Cardiology 2015 Congress. London (2015).

69. Cappato R, Marchlinski FE, Hohnloser SH, et al. Uninterrupted rivaroxaban vs. uninterrupted vitamin $\mathrm{K}$ antagonists for catheter ablation in non-valvular atrial fibrillation. Eur Heart J. 2015;36(28):1805-11.

70. Pathak RK, Middeldorp ME, Meredith M, et al. Long-term effect of goal-directed weight management in an atrial fibrillation cohort: a long-term follow-up study (LEGACY). J Am Coll Cardiol. 2015;65(20):2159-69.

71. Nichol G, Leroux B, Wang $H$, et al. Trial of continuous or interrupted chest compressions during CPR. N Engl J Med. 2015;373(23):2203-14.

72. Douglas PS, Hoffmann U, Patel MR, et al. Outcomes of anatomical versus functional testing for coronary artery disease. N Engl J Med. 2015;372(14): 1291-300.

73. Newby D, Williams M, Hunter A, The SCOT-HEART investigators, et al. CT coronary angiography in patients with suspected angina due to coronary heart disease (SCOT-HEART): an open-label, parallel-group, multicentre trial. Lancet 2015;385(9985): 2383-91. 